\title{
An identity based on the generalised negative binomial distribution with applications in ruin theory
}

\author{
David C M Dickson \\ Centre for Actuarial Studies, Department of Economics, \\ University of Melbourne, VIC 3010, Australia \\ dcmd@unimelb.edu.au
}

\begin{abstract}
In this note we show how expressions for the probability of ultimate ruin can be obtained from the probability function of the time of ruin in a particular compound binomial risk model, and from the density of the time of ruin in a particular Sparre Andersen risk model. In each case evaluation of generalised binomial series is required, and the argument of each series has a common form. We evaluate these series by creating an identity based on the generalised negative binomial distribution. We also show how the same ideas apply to the probability function of the number of claims in a particular Sparre Andersen model.
\end{abstract}

Keywords: generalised negative binomial distribution, generalised binomial series, ruin probability, time of ruin, Erlang distribution

\section{Introduction}

Certain identities in ruin theory are trivially obvious in general, but are more difficult to obtain in specific cases. For example, in a Sparre Andersen risk model, if $\psi(u)$ denotes the probability of ultimate ruin from initial surplus $u$, and $w(u, t)$ for $t>0$ denotes the defective density of the time of ruin given initial surplus $u$, then it is true that $\psi(u)=\int_{0}^{\infty} w(u, t) d t$. What is perhaps less obvious is how to obtain an expression for $\psi(u)$ given an expression for $w(u, t)$. This is the type of question we consider in this note. Our objective is not to derive new results in ruin theory. Rather, we aim to show how a particular identity, discussed in Section 3, allows us to obtain identities that we know must hold from general considerations.

Recent papers by the author, e.g. Dickson and Li (2010) and Dickson (2012), make use of generalised binomial series in solving finite time ruin problems, and these will be central in what follows. A generalised binomial series is defined by Graham et al. (1994) as

$$
\mathcal{B}_{\tau}(z)=\sum_{k=0}^{\infty}\left(\begin{array}{c}
\tau k+1 \\
k
\end{array}\right) \frac{z^{k}}{\tau k+1}
$$


and this series has the extremely useful property that

$$
\mathcal{B}_{\tau}(z)^{r}=\sum_{k=0}^{\infty}\left(\begin{array}{c}
\tau k+r \\
k
\end{array}\right) \frac{r z^{k}}{\tau k+r} .
$$

Unfortunately, for most values of $\tau$ a simple form of $\mathcal{B}_{\tau}(z)$ does not exist, a notable exception being the case $\tau=2$, with

$$
\mathcal{B}_{2}(z)=\frac{1-\sqrt{1-4 z}}{2 z}
$$

See Graham et al. (1994). Equation (1.1) has been used in relation to problems involving the number of claims until ruin in the classical risk model and variations thereof; see Dickson (2012) and Dickson and Qazvini (2016). Generalised binomial series with integer values of $\tau$ greater than 2 appear in other ruin problems, and in this note we show how such series can be evaluated. Evaluation hinges on the fact that if we can write $z$ in a particular way that occurs in ruin problems, then we can obtain a simple expression for $\mathcal{B}_{\tau}(z)$.

\section{Preliminaries}

In this section we describe the risk models discussed in this note, and as the models are well-known, the descriptions are brief. The first is the compound binomial model introduced by Gerber (1988). This is a discrete time model where the surplus process $\left\{U_{d}(n)\right\}_{n=0}^{\infty}$ is given by $U_{d}(n)=u+n-\sum_{i=1}^{n} X_{i}$ with $U_{d}(0)=u$, where $u$ is a non-negative integer. The premium income per unit time is 1 and $\left\{X_{i}\right\}_{i=1}^{\infty}$ is a sequence of independent and identically distributed (i.i.d.) random variables, distributed on the non-negative integers, and representing aggregate claims per unit time. The ultimate ruin probability for this model can be defined in two different ways. The definition used in this note is that used by $\mathrm{Li}$ and Sendova (2013), namely

$$
\psi_{d}(u)=\operatorname{Pr}\left(U_{d}(n)<0 \quad \text { for some } n, n=1,2,3, \ldots\right),
$$

but we could equally have used the definition given by Gerber (1988) and reworked the results we use below from $\mathrm{Li}$ and Sendova (2013). The net profit condition for this model is $E\left[X_{1}\right]<1$. When this condition holds, $\psi_{d}(u)<1$, and when it does not, $\psi_{d}(u)=1$. The time of ruin is defined as $T_{d, u}=\min \left\{n \geq 1: U_{d}(n)<0\right\}$, and the probability function of the time of ruin is $w_{d}(u, n)=\operatorname{Pr}\left(T_{d, u}=n\right)$ for $n=1,2,3, \ldots$.

The second model we consider is the model introduced by Sparre Andersen (1957). Let $\left\{W_{i}\right\}_{i=1}^{\infty}$ be a sequence of i.i.d. random variables representing the times between claims (with $W_{1}$ being the time to the first claim), and let $\left\{Y_{i}\right\}_{i=1}^{\infty}$ be a sequence of i.i.d. random variables representing the amounts of the individual claims. The premium income per unit time is $c>0$ and the surplus immediately following payment of the $n$th claim, $n=1,2,3, \ldots$, is $U(n)=u+\sum_{i=1}^{n}\left(c W_{i}-Y_{i}\right)$ where $u \geq 0$ is the initial surplus. The ultimate ruin probability is defined as 
$\psi(u)=\operatorname{Pr}(U(n)<0 \quad$ for some $n, n=1,2,3, \ldots)$. The net profit condition for this model is $c>E\left[Y_{1}\right] / E\left[W_{1}\right]$, and when this condition holds $\psi(u)<1$. If the net profit condition does not hold, we have $\psi(u)=1$. The time of ruin is defined as $T_{u}=\inf \{t: U(t)<0\}$, and the defective density of the time of ruin is denoted $w(u, t)$ for $t>0$ where $w(u, t)=\frac{d}{d t} \operatorname{Pr}\left(T_{u} \leq t\right)$.

The simplest version of the Sparre Anderson model is the classical risk model in which the distribution of $W_{1}$ is exponential, meaning that claim arrivals are modelled as a Poisson process.

We now state two results which feature later in this note. First, in a Sparre Andersen model for which the net profit condition holds and the individual claim amount distribution is exponential with mean $1 / \alpha$,

$$
\psi(u)=(1-R / \alpha) e^{-R u}
$$

where $R$ is the adjustment coefficient, and is the unique positive number satisfying $E\left[e^{-c R W_{1}}\right] E\left[e^{R Y_{1}}\right]=1$. See, for example, Grandell (1991). When the distribution of $W_{1}$ is exponential with parameter $\lambda, R=\alpha-\lambda / c$. Second, in the case when the distribution of $W_{1}$ is exponential with parameter $\lambda$ and the net profit condition holds, $\psi(0)=\lambda E\left[Y_{1}\right] / c$. See, for example, Grandell (1991).

Most of this note involves Erlang distributions. That is because few explicit results exist for finite time ruin problems, and most of these for Sparre Andersen models involve Erlang distributions, either as the distribution for inter-claim times or for individual claim amounts. See Landriault et al. (2011) and references therein.

\section{A useful identity}

The generalised negative binomial distribution was introduced by Jain and Consul (1971). A generalised negative binomial random variable, $N$, has a probability function defined in terms of parameters $k, p$ and $\beta$ as

$$
\operatorname{Pr}(N=n)=\left(\begin{array}{c}
\beta n+k \\
n
\end{array}\right) \frac{k}{\beta n+k} p^{n}(1-p)^{n(\beta-1)+k}
$$

for $n=0,1,2, \ldots$, where $k>0,0<p<1$ and $|\beta p|<1$. As the sum over $n$ of this probability function is 1 , it follows that

$$
(1-p)^{-k}=\sum_{n=0}^{\infty}\left(\begin{array}{c}
\beta n+k \\
n
\end{array}\right) \frac{k}{\beta n+k} p^{n}(1-p)^{n(\beta-1)}=\left(\mathcal{B}_{\beta}\left(p(1-p)^{\beta-1}\right)\right)^{k}
$$

giving the useful identity

$$
\mathcal{B}_{\beta}\left(p(1-p)^{\beta-1}\right)=(1-p)^{-1} .
$$

In everything that follows, $\beta$ is a positive integer at least equal to 2 , and equation (3.1) offers a means of evaluating generalised binomial series. However, we need to be careful with the application of (3.1). Consider the function $f(p)=p(1-p)^{\beta-1}$ 
over the interval $[0,1]$. We have $f(0)=f(1)=0$, and the function has a unique maximum at $p=1 / \beta$. So, if we can actually write $z=p(1-p)^{\beta-1}$ in equation (3.1), then there will be two solutions for $p$ unless we are writing $z$ in terms of the value of $p$ that maximises $f(p)$.

In Sections 4 to 6 we can make use of each solution. Only one solution is really of interest, and that solution corresponds to the case when the net profit condition holds for the risk model we consider. We will see that the condition

$$
|\beta p|<1
$$

on the parameters of the generalised negative binomial distribution tells us which of the two solutions to select.

When $p=1 / \beta$ then (3.1) still holds, even though the condition (3.2) does not, with

$$
\mathcal{B}_{\beta}\left(\frac{(\beta-1)^{\beta-1}}{\beta^{\beta}}\right)=\frac{\beta}{\beta-1} .
$$

This follows from the first identity in (5.59) of Graham et al. (1994). This is not a particularly significant result in the context of ruin problems, but we state it because in all the applications of (3.1) that follow, the case $p=1 / \beta$ corresponds to the premium equalling expected claims. In the case of two solutions, one solution corresponds to the premium exceeding expected claims, the other to the premium being less than expected claims.

\section{The compound binomial model}

In this section we give a simple illustration of the usefulness of equation (3.1). Li and Sendova (2013) show that the probability function of the time of ruin when aggregate claims are geometrically distributed with probability function $(1-q) q^{x}$ for $x=0,1,2, \ldots$ is

$$
w_{d}(u, n)=q^{n+u+1}(1-q)^{n-1} \frac{u+2}{2 n+u}\left(\begin{array}{c}
2 n+u \\
n-1
\end{array}\right)
$$

for $n=1,2,3, \ldots$. They also show that when the net profit condition holds, i.e. $q<1 / 2, \psi_{d}(u)=(q /(1-q))^{u+2}$ for $u=0,1,2, \ldots$ We can obtain this result from their expression for $w_{d}(u, n)$ as

$$
\begin{aligned}
\psi_{d}(u) & =\sum_{n=1}^{\infty} w_{d}(u, n)=\sum_{m=0}^{\infty} q^{m+u+2}(1-q)^{m} \frac{u+2}{2 m+u+2}\left(\begin{array}{c}
2 m+u+2 \\
m
\end{array}\right) \\
& =q^{u+2} \mathcal{B}_{2}(q(1-q))^{u+2} .
\end{aligned}
$$

When $q<1 / 2$ the net profit condition holds, and condition (3.2) is satisfied (with $\beta=2$ and $p=q$ ), so $\mathcal{B}_{2}(q(1-q))^{u+2}=(1-q)^{-(u+2)}$ and hence $\psi_{d}(u)=$ $(q /(1-q))^{u+2}$.

When $q>1 / 2$ the net profit condition does not hold, nor is condition (3.2) satisfied, so $\mathcal{B}_{2}(q(1-q))^{u+2}=q^{-(u+2)}$ and hence $\psi_{d}(u)=1$. The same conclusion holds when $q=1 / 2$. 
Although formula (1.1) gives us an expression for $\mathcal{B}_{2}(z)$, we did not need it. Our approach gives the solution in the case of interest, i.e. the net profit condition holds, as well as giving the solution when it does not.

\section{$5 \quad \operatorname{Erlang}(n)$ risk model with exponential claims}

In this section we consider an Erlang risk model with exponential claims. Specifically, we assume that $W_{1} \sim \operatorname{Erl}(n, \lambda)$ so that $E\left(W_{1}\right)=n / \lambda$ and $Y_{1} \sim \operatorname{Erl}(1, \alpha)$. We consider two formulae for the density of the time of ruin and explain how the ultimate ruin probability can be derived from each.

\subsection{The density of the time of ruin}

We consider first a formula which is implicit in the literature, namely

$$
w(u, t)=e^{-\alpha u-(\lambda+\alpha c) t} \sum_{j=1}^{\infty} \frac{(\alpha u)^{j-1}(\lambda t)^{n j}(n j)}{(j-1) !} \sum_{m=0}^{\infty} \frac{\left(\alpha c \lambda^{n}\right)^{m} t^{m(n+1)-1}}{m ![n(m+j)] !} .
$$

This formula is obtained from results stated in Nie et al. (2015). Special cases of this formula have been derived previously, by Drekic and Willmot (2003) in the case $n=1$, and by Dickson and Li (2010) in the case $n=2$. We start with this formula for $w(u, t)$ as the manipulations are more straightforward than those in the next subsection. We now give the main result of this subsection.

Theorem 5.1 The ultimate ruin probability is

$$
\psi(u)=\left(\frac{\lambda \mathcal{B}_{n+1}\left(Q_{n}\right)}{\lambda+\alpha c}\right)^{n} \exp \left\{-\alpha u\left(1-\left(\frac{\lambda \mathcal{B}_{n+1}\left(Q_{n}\right)}{\lambda+\alpha c}\right)^{n}\right)\right\}
$$

where $Q_{n}=\alpha c \lambda^{n} /(\lambda+\alpha c)^{n+1}$.

Proof 5.1 Integrating expression (5.1) with respect to $t$ over $(0, \infty)$ gives $\psi(u)$, and thus

$$
\begin{aligned}
\psi(u) & =e^{-\alpha u} \sum_{j=1}^{\infty} \frac{(\alpha u)^{j-1} \lambda^{n j}(n j)}{(j-1) !} \sum_{m=0}^{\infty} \frac{\left(\alpha c \lambda^{n}\right)^{m} \Gamma(n j+m(n+1))}{m ![n(m+j)] !(\lambda+\alpha c)^{n j+m(n+1)}} \\
& =e^{-\alpha u} \sum_{j=1}^{\infty} \frac{(\alpha u)^{j-1} \lambda^{n j}}{(j-1) !(\lambda+\alpha c)^{n j}} \sum_{m=0}^{\infty}\left(\begin{array}{c}
(n+1) m+n j \\
m
\end{array}\right) \frac{n j Q_{n}^{m}}{(n+1) m+n j} .
\end{aligned}
$$

As

$$
\sum_{m=0}^{\infty}\left(\begin{array}{c}
(n+1) m+n j \\
m
\end{array}\right) \frac{n j Q_{n}^{m}}{(n+1) m+n j}=\mathcal{B}_{n+1}\left(Q_{n}\right)^{n j}
$$

it follows that

$$
\begin{aligned}
\psi(u) & =e^{-\alpha u} \sum_{j=1}^{\infty} \frac{(\alpha u)^{j-1} \lambda^{n j} \mathcal{B}_{n+1}\left(Q_{n}\right)^{n j}}{(j-1) !(\lambda+\alpha c)^{n j}} \\
& =\left(\frac{\lambda \mathcal{B}_{n+1}\left(Q_{n}\right)}{\lambda+\alpha c}\right)^{n} \exp \left\{-\alpha u\left(1-\left(\frac{\lambda \mathcal{B}_{n+1}\left(Q_{n}\right)}{\lambda+\alpha c}\right)^{n}\right)\right\} .
\end{aligned}
$$


Before we explain how equation (3.1) can be used to evaluate the generalised binomial series in (5.2) we remark that from formula (2.1), when the net profit condition holds the adjustment coefficient is given by

$$
R=\alpha\left(1-\left(\frac{\lambda \mathcal{B}_{n+1}\left(Q_{n}\right)}{\lambda+\alpha c}\right)^{n}\right)
$$

\subsubsection{The case $n=1$}

When $n=1$, our risk model is the classical risk model. If we first make the usual assumption that the net profit condition holds, i.e. $c>\lambda / \alpha$, then from (3.1) with $\beta=2$ we see that the condition $|2 p|<1$ leads to the choice $p=\lambda /(\lambda+\alpha c)$. Hence $\mathcal{B}_{2}\left(Q_{1}\right)=(\lambda+\alpha c) /(\alpha c)$, formula (5.2) gives the familiar result

$$
\psi(u)=\frac{\lambda}{\alpha c} \exp \{-(\alpha-\lambda / c) u\}
$$

and formula (5.4) gives $R=\alpha-\lambda / c$. However, if we make the assumption that $0<c \leq \lambda / \alpha$, then the net profit condition does not hold, and we see that the correct choice is $p=\alpha c /(\lambda+\alpha c)$. In this case $\mathcal{B}_{2}\left(Q_{1}\right)=(\lambda+\alpha c) / \lambda$, leading to $\psi(u)=1$.

\subsubsection{The case $n>1$}

When the net profit condition $c>\lambda /(n \alpha)$ holds, condition (3.1) is satisfied by $\tilde{p}<1 /(n+1)$ such that

$$
\tilde{p}(1-\tilde{p})^{n}=\alpha c \lambda^{n} /(\lambda+\alpha c)^{n+1} .
$$

Then

$$
\left(\frac{\lambda \mathcal{B}_{n+1}\left(Q_{n}\right)}{\lambda+\alpha c}\right)^{n}=\left(\frac{\lambda}{(\lambda+\alpha c)(1-\tilde{p})}\right)^{n}=\frac{(\lambda+\alpha c) \tilde{p}}{\alpha c}=\psi(0),
$$

and we see that $\psi(u)=\psi(0) \exp \{-R u\}$ with $R=\alpha-\tilde{p}(\alpha+\lambda / c)$.

In the case when $0<c<\lambda /(n \alpha)$, condition (3.1) is satisfied by $p=\alpha c /(\lambda+\alpha c)$, resulting in $B_{n+1}\left(Q_{n}\right)=(\lambda+\alpha c) / \lambda$, which gives $\psi(u)=1$. The same conclusion is reached when $c=\lambda /(n \alpha)$.

\section{$5.2 \quad$ A second identity}

Dickson et al. (2005) obtain a different formula for $w(u, t)$, and they integrate this formula to obtain an expression for $\psi(u)$. We now show that this expression leads to the same results for $\psi(u)$ as in the previous subsection. To do this we make use of identity (5.61) from Graham et al. (1994), namely

$$
\sum_{k=0}^{\infty}\left(\begin{array}{c}
\tau k+r \\
k
\end{array}\right) z^{k}=\frac{\mathcal{B}_{\tau}(z)^{r}}{1-\tau+\tau \mathcal{B}_{\tau}(z)^{-1}}
$$


Starting from formula (3.12) of Dickson et al. (2005), and with the notation $(a)_{n}=$ $\Gamma(n+a) / \Gamma(a)$ (Pochhammer's symbol), we have

$$
\begin{aligned}
& \psi(u)=e^{-\alpha u} \sum_{m=0}^{\infty}\left(\frac{\lambda}{\lambda+\alpha c}\right)^{n m+n} \sum_{j=0}^{m} \frac{(n m+n)_{j}}{j !(m-j) !}\left(\frac{\alpha c}{\lambda+\alpha c}\right)^{j}(\alpha u)^{m-j} \\
& -\frac{\alpha c n e^{-\alpha u}}{\lambda+\alpha c} \sum_{m=0}^{\infty}\left(\frac{\lambda}{\lambda+\alpha c}\right)^{n m+2 n} \sum_{j=0}^{m} \frac{(n m+2 n+1)_{j}}{j !(m-j) !}\left(\frac{\alpha c}{\lambda+\alpha c}\right)^{j}(\alpha u)^{m-j} \\
& =e^{-\alpha u} \sum_{m=0}^{\infty} \sum_{j=0}^{\infty}\left(\frac{\lambda}{\lambda+\alpha c}\right)^{n(m+j)+n} \frac{(n(m+j)+n)_{j}}{j ! m !}\left(\frac{\alpha c}{\lambda+\alpha c}\right)^{j}(\alpha u)^{m} \\
& -\frac{\alpha c n e^{-\alpha u}}{\lambda+\alpha c} \sum_{m=0}^{\infty} \sum_{j=0}^{\infty}\left(\frac{\lambda}{\lambda+\alpha c}\right)^{n(m+j)+2 n} \frac{(n(m+j)+2 n+1)_{j}}{j ! m !}\left(\frac{\alpha c}{\lambda+\alpha c}\right)^{j}(\alpha u)^{m} \\
& =e^{-\alpha u} \sum_{m=0}^{\infty}\left(\frac{\lambda}{\lambda+\alpha c}\right)^{n m+n} \frac{(\alpha u)^{m}}{m !} \sum_{j=0}^{\infty} \frac{\Gamma(n(m+j)+n+j)}{j ! \Gamma(n(m+j)+n)} Q_{n}^{j} \\
& -\frac{\alpha c n e^{-\alpha u}}{\lambda+\alpha c} \sum_{m=0}^{\infty}\left(\frac{\lambda}{\lambda+\alpha c}\right)^{n m+2 n} \frac{(\alpha u)^{m}}{m !} \sum_{j=0}^{\infty} \frac{\Gamma(n(m+j)+2 n+1+j)}{j ! \Gamma(n(m+j)+2 n+1)} Q_{n}^{j}
\end{aligned}
$$

where $Q_{n}=\alpha c \lambda^{n} /(\lambda+\alpha c)^{n+1}$ as before. Note that we have used the identity $\sum_{m=0}^{\infty} \sum_{j=0}^{m} g(m, j)=\sum_{m=0}^{\infty} \sum_{j=0}^{\infty} g(m+j, j)$ in the first step. Now the sum over $j$ in (5.8) can be written as

$$
\sum_{j=0}^{\infty}\left(\begin{array}{c}
(n+1) j+n(m+1)-1 \\
j
\end{array}\right) Q_{n}^{j}
$$

and from (5.7) this can be written as

$$
\frac{\mathcal{B}_{n+1}\left(Q_{n}\right)^{n(m+1)-1}}{(n+1) \mathcal{B}_{n+1}\left(Q_{n}\right)^{-1}-n}
$$

and similarly the sum over $j$ in $(5.9)$ is

$$
\sum_{j=0}^{\infty}\left(\begin{array}{c}
(n+1) j+n(m+2) \\
j
\end{array}\right) Q_{n}^{j}=\frac{\mathcal{B}_{n+1}\left(Q_{n}\right)^{n(m+2)}}{(n+1) \mathcal{B}_{n+1}\left(Q_{n}\right)^{-1}-n}
$$

So we get

$$
\begin{aligned}
\psi(u)= & \frac{e^{-\alpha u}\left(\frac{\lambda}{\lambda+\alpha c}\right)^{n} \mathcal{B}_{n+1}\left(Q_{n}\right)^{n-1}}{(n+1) \mathcal{B}_{n+1}\left(Q_{n}\right)^{-1}-n} \sum_{m=0}^{\infty} \frac{\left(\alpha u\left(\frac{\lambda}{\lambda+\alpha c}\right)^{n}\right)^{m} \mathcal{B}_{n+1}\left(Q_{n}\right)^{n m}}{m !} \\
& -\frac{\alpha c n\left(\frac{\lambda}{\lambda+\alpha c}\right)^{2 n} \mathcal{B}_{n+1}\left(Q_{n}\right)^{2 n} e^{-\alpha u}}{\lambda+\alpha c} \sum_{m=0}^{\infty} \frac{\left(\alpha u\left(\frac{\lambda}{\lambda+\alpha c}\right)^{n}\right)^{m} \mathcal{B}_{n+1}\left(Q_{n}\right)^{n m}}{m !} \\
= & \frac{\left(\frac{\lambda}{\lambda+\alpha c}\right)^{n} \mathcal{B}_{n+1}\left(Q_{n}\right)^{n-1}}{(n+1) \mathcal{B}_{n+1}\left(Q_{n}\right)^{-1}-n} \exp \left\{-\alpha u\left(1-\left(\frac{\lambda \mathcal{B}_{n+1}\left(Q_{n}\right)}{\lambda+\alpha c}\right)^{n}\right)\right\}
\end{aligned}
$$




$$
-\frac{\alpha c n\left(\frac{\lambda}{\lambda+\alpha c}\right)^{2 n} \mathcal{B}_{n+1}\left(Q_{n}\right)^{2 n}}{(\lambda+\alpha c)\left((n+1) \mathcal{B}_{n+1}\left(Q_{n}\right)^{-1}-n\right)} \exp \left\{-\alpha u\left(1-\left(\frac{\lambda \mathcal{B}_{n+1}\left(Q_{n}\right)}{\lambda+\alpha c}\right)^{n}\right)\right\}
$$

giving

$$
\psi(u)=\psi(0) \exp \left\{-\alpha u\left(1-\left(\frac{\lambda \mathcal{B}_{n+1}\left(Q_{n}\right)}{\lambda+\alpha c}\right)^{n}\right)\right\}
$$

with

$$
\begin{aligned}
\psi(0) & =\frac{\left(\frac{\lambda}{\lambda+\alpha c}\right)^{n} \mathcal{B}_{n+1}\left(Q_{n}\right)^{n-1}}{(n+1) \mathcal{B}_{n+1}\left(Q_{n}\right)^{-1}-n}\left(1-\frac{\alpha c n\left(\frac{\lambda}{\lambda+\alpha c}\right)^{n} \mathcal{B}_{n+1}\left(Q_{n}\right)^{n+1}}{\lambda+\alpha c}\right) \\
& =\frac{\left(\frac{\lambda}{\lambda+\alpha c}\right)^{n} \mathcal{B}_{n+1}\left(Q_{n}\right)^{n}}{n+1-n \mathcal{B}_{n+1}\left(Q_{n}\right)}\left(1-n Q_{n} \mathcal{B}_{n+1}\left(Q_{n}\right)^{n+1}\right) .
\end{aligned}
$$

From the first identity in (5.59) of Graham et al. (1994) we know that

$$
Q_{n} \mathcal{B}_{n+1}\left(Q_{n}\right)^{n+1}=\mathcal{B}_{n+1}\left(Q_{n}\right)-1,
$$

giving

$$
1-n Q_{n} \mathcal{B}_{n+1}\left(Q_{n}\right)^{n+1}=n+1-n \mathcal{B}_{n+1}\left(Q_{n}\right),
$$

and so, as before, $\psi(0)=\left(\lambda \mathcal{B}_{n+1}\left(Q_{n}\right) /(\lambda+\alpha c)\right)^{n}$ and formula $(5.10)$ for $\psi(u)$ is the same as (5.2).

\section{The number of claims until ruin}

In this section we consider the number of claims until ruin from initial surplus $u$, denoted by $N_{u}$, with probability function $p_{k}(u)=\operatorname{Pr}\left(N_{u}=k\right)$. Our aim here is to illustrate how identity (3.1) allows us to show that $\sum_{k=1}^{\infty} p_{k}(u)=\psi(u)$ in particular cases. We start by considering the model of the previous section, then consider an identity under the classical risk model.

\section{1 $\quad$ Erlang $(n)$ risk model}

For the model considered in Section 5, Frostig et al. (2012) show that

$$
p_{k}(0)=\frac{\lambda^{n k}(\alpha c)^{k-1}[(n+1) k-2] !}{(\lambda+\alpha c)^{(n+1) k-1}(n k-1) ! k !}
$$

for $k=1,2,3, \ldots$ (See also Landriault et al. (2011) and Zhao and Zhang (2012).) We now generalise this result to the case $u>0$, and the key reason for taking the following approach is that our proof also shows why $\sum_{k=1}^{\infty} p_{k}(u)=\psi(u)$. From arguments in Dickson and Qazvini (2016) concerning the classical risk model with exponential claims, we can easily show that the probability generating function of $N_{u}$ for this model is

$$
P_{N_{u}}(r)=E\left[r^{N_{u}} I\left(T_{u}<\infty\right)\right]=\sum_{m=1}^{\infty} \frac{(\alpha u)^{m-1} e^{-\alpha u}}{(m-1) !} P_{N_{0}}(r)^{m},
$$


where $I$ is the indicator function. So, by deriving $P_{N_{0}}(r)$ we can find $P_{N_{u}}(r)$, and we do this in the next two theorems. Before doing so, we remark that Landriault et al. (2011) obtain an expression (their formula (23)) for the probability function of the number of claims until ruin in a Sparre Andersen model where the inter-claim time distribution is an infinite mixture of Erlang distributions with the same scale parameter and the claim size distribution is exponential. Formula (6.4) below for $p_{m}(u)$ is new, although it can also be obtained by manipulating formula (23) of Landriault et al. (2011). We do not consider the more general probability function obtained by Landiault et al. (2011) because, as far as we can tell, summation of it does not lead to generalised binomial series other than in the special case that a single mixing weight is 1, i.e. the case we consider of the Erlang distribution.

Theorem 6.1 The probability generating function of $N_{0}$ is

$$
P_{N_{0}}(r)=\frac{r \lambda^{n}}{(\lambda+\alpha c)^{n}} \mathcal{B}_{n+1}\left(\frac{\alpha c r \lambda^{n}}{(\lambda+\alpha c)^{n+1}}\right)^{n}
$$

Proof 6.1 Using expression (6.1) we have

$$
\begin{aligned}
P_{N_{0}}(r) & =\sum_{k=1}^{\infty} r^{k} p_{k}(0)=\sum_{k=1}^{\infty} \frac{\left(r \lambda^{n}\right)^{k}(\alpha c)^{k-1}[(n+1) k-2] !}{(\lambda+\alpha c)^{(n+1) k-1}(n k-1) ! k !} \\
& =\sum_{t=0}^{\infty} \frac{\left(r \lambda^{n}\right)^{t+1}(\alpha c)^{t}[(n+1)(t+1)-2] !}{(\lambda+\alpha c)^{(n+1)(t+1)-1}[n(t+1)-1] !(t+1) !} \\
& =\frac{r \lambda^{n}}{(\lambda+\alpha c)^{n}} \sum_{t=0}^{\infty}\left(\frac{\alpha c r \lambda^{n}}{(\lambda+\alpha c)^{n+1}}\right)^{t} \frac{(n t+t+n-1) !}{[n(t+1)-1] !(t+1) !} \\
& =\frac{r \lambda^{n}}{(\lambda+\alpha c)^{n}} \sum_{t=0}^{\infty} \frac{[(n+1) t+n] !}{[n(t+1)-1] !(t+1) !} \frac{1}{(n+1) t+n}\left(\frac{\alpha c r \lambda^{n}}{(\lambda+\alpha c)^{n+1}}\right)^{t} .
\end{aligned}
$$

Now the sum in the previous line is $\mathcal{B}_{n+1}\left(\alpha c r \lambda^{n} /(\lambda+\alpha c)^{n+1}\right)^{n}$ if

$$
\frac{[(n+1) t+n] !}{[n(t+1)-1] !(t+1) !}=n\left(\begin{array}{c}
(n+1) t+n \\
t
\end{array}\right)
$$

and this is easily seen to be an equality, giving (6.3).

Theorem 6.2 For $m=1,2,3, \ldots$,

$$
p_{m}(u)=e^{-\alpha u}\left(\frac{\lambda}{\lambda+\alpha c}\right)^{n m} \sum_{k=0}^{m-1}\left(\frac{\alpha c}{\lambda+\alpha c}\right)^{k} \frac{(\alpha u)^{m-k-1}}{(m-k-1) !}\left(\begin{array}{c}
n m+k \\
k
\end{array}\right) \frac{n(m-k)}{n m+k} .
$$

Proof 6.2 Starting from expression (6.2) and inserting expression (6.3) for $P_{N_{0}}(r)$ we have

$$
P_{N_{u}}(r)=\sum_{m=1}^{\infty} \frac{(\alpha u)^{m-1} e^{-\alpha u}}{(m-1) !}\left(\frac{r \lambda^{n}}{(\lambda+\alpha c)^{n}}\right)^{m} \mathcal{B}_{n+1}\left(\frac{\alpha c r \lambda^{n}}{(\lambda+\alpha c)^{n+1}}\right)^{m n}
$$




$$
\begin{gathered}
=\sum_{m=1}^{\infty} \sum_{t=0}^{\infty} \frac{(\alpha u)^{m-1} e^{-\alpha u}}{(m-1) !}\left(\frac{r \lambda^{n}}{(\lambda+\alpha c)^{n}}\right)^{m}\left(\begin{array}{c}
(n+1) t+m n \\
t
\end{array}\right) \\
\times\left(\frac{\alpha c r \lambda^{n}}{(\lambda+\alpha c)^{n+1}}\right)^{t} \frac{m n}{(n+1) t+m n} .
\end{gathered}
$$

We now use the identity

$$
\sum_{m=1}^{\infty} \sum_{t=0}^{\infty} g(m, t)=\sum_{m=1}^{\infty} \sum_{t=0}^{m-1} g(t+1, m-t-1)
$$

for some function $g$ to get

$$
\begin{aligned}
P_{N_{u}}(r)=e^{-\alpha u} \sum_{m=1}^{\infty} & \left(\frac{r \lambda^{n}}{(\lambda+\alpha c)^{n}}\right)^{m} \sum_{t=0}^{m-1}\left(\frac{\alpha c}{\lambda+\alpha c}\right)^{m-t-1} \frac{(\alpha u)^{t}}{t !} \\
& \times\left(\begin{array}{c}
(n+1) m-t-1 \\
m-t-1
\end{array}\right) \frac{n(t+1)}{(n+1) m-t-1} \\
=e^{-\alpha u} \sum_{m=1}^{\infty} & \left(\frac{r \lambda^{n}}{(\lambda+\alpha c)^{n}}\right)^{m} \sum_{k=0}^{m-1}\left(\frac{\alpha c}{\lambda+\alpha c}\right)^{k} \frac{(\alpha u)^{m-k-1}}{(m-k-1) !} \\
& \times\left(\begin{array}{c}
n m+k \\
k
\end{array}\right) \frac{n(m-k)}{n m+k} .
\end{aligned}
$$

As the coefficient of $r^{m}$ in (6.7) is $p_{m}(u)$ we obtain

$$
p_{m}(u)=e^{-\alpha u}\left(\frac{\lambda}{\lambda+\alpha c}\right)^{n m} \sum_{k=0}^{m-1}\left(\frac{\alpha c}{\lambda+\alpha c}\right)^{k} \frac{(\alpha u)^{m-k-1}}{(m-k-1) !}\left(\begin{array}{c}
n m+k \\
k
\end{array}\right) \frac{n(m-k)}{n m+k} .
$$

It is straightforward to show that $\sum_{m=1}^{\infty} p_{m}(u)=\psi(u)$ because all we have to do is set $r=1$ in (6.7) and then work backwards through the proof of Theorem 6.2 to (6.5). The only difficult step without knowing the derivation of the probability function is to apply identity (6.6). The equation we obtain for $\psi(u)$ is the same as (5.2) since when $r=1$ expressions (6.5) and (5.3) are identical.

\subsection{Classical risk model}

For the classical risk model, a case other than that of exponential claims in which we can find an explicit form for $p_{n}(u)$ is when $u=0$ and individual claims are $\operatorname{Erl}(m, \mu)$ distributed. In this case we can show from formulae (6) and (7) of Dickson (2012) that

$$
p_{n}(0)=\sum_{i=0}^{m-1} \frac{(m(n-1)+n+i-1) !(i+1)}{(n-1) !(m(n-1)+i+1) !} \frac{\lambda^{n}(\mu c)^{m(n-1)+i}}{(\lambda+\mu c)^{m(n-1)+n+i}} .
$$

We can use this to obtain $\psi(0)$ since

$$
\sum_{n=1}^{\infty} p_{n}(0)=\sum_{n=1}^{\infty} \sum_{i=0}^{m-1} \frac{(m(n-1)+n+i-1) !(i+1)}{(n-1) !(m(n-1)+i+1) !} \frac{\lambda^{n}(\mu c)^{m(n-1)+i}}{(\lambda+\mu c)^{m(n-1)+n+i}}
$$




$$
\begin{aligned}
& =\sum_{i=0}^{m-1} \frac{(i+1)(\mu c)^{i}}{(\lambda+\mu c)^{i}} \sum_{n=1}^{\infty} \frac{(m(n-1)+n+i-1) !}{(n-1) !(m(n-1)+i+1) !} \frac{\lambda^{n}(\mu c)^{m(n-1)}}{(\lambda+\mu c)^{m(n-1)+n}} \\
& =\sum_{i=0}^{m-1} \frac{(i+1)(\mu c)^{i-m}}{(\lambda+\mu c)^{i-m}} \sum_{n=1}^{\infty} \frac{(m(n-1)+n+i) !}{(n-1) !(m(n-1)+i+1) !} \frac{\tilde{Q}_{m}^{n}}{m(n-1)+n+i}
\end{aligned}
$$

where $\tilde{Q}_{m}=\lambda(\mu c)^{m} /(\lambda+\mu c)^{m+1}$. Then

$$
\begin{aligned}
\sum_{n=1}^{\infty} p_{n}(0) & =\sum_{i=0}^{m-1} \frac{(i+1)(\mu c)^{i-m}}{(\lambda+\mu c)^{i-m}} \sum_{r=0}^{\infty}\left(\begin{array}{c}
m r+r+i+1 \\
r
\end{array}\right) \frac{\tilde{Q}_{m}^{r+1}}{m r+r+i+1} \\
& =\tilde{Q}_{m} \sum_{i=0}^{m-1} \frac{(\mu c)^{i-m}}{(\lambda+\mu c)^{i-m}} \sum_{r=0}^{\infty}\left(\begin{array}{c}
r(m+1)+i+1 \\
r
\end{array}\right) \frac{(i+1) \tilde{Q}_{m}^{r}}{r(m+1)+i+1} \\
& =\tilde{Q}_{m} \sum_{i=0}^{m-1} \frac{(\mu c)^{i-m}}{(\lambda+\mu c)^{i-m}} \mathcal{B}_{m+1}\left(\tilde{Q}_{m}\right)^{i+1} \\
& =\frac{\lambda \mathcal{B}_{m+1}\left(\tilde{Q}_{m}\right)}{\lambda+\mu c} \sum_{i=0}^{m-1}\left(\frac{\mu c \mathcal{B}_{m+1}\left(\tilde{Q}_{m}\right)}{\mu c+\lambda}\right)^{i}
\end{aligned}
$$

The net profit condition is $c>\lambda m / \mu$, and when this holds we see from (3.2) with $\beta=m+1$ that $p=\lambda /(\mu c+\lambda)$ and so

$$
\mathcal{B}_{m+1}\left(\tilde{Q}_{m}\right)=(\lambda+\mu c) /(\mu c) .
$$

Then from (6.8) we obtain the expected result

$$
\sum_{n=1}^{\infty} p_{n}(0)=\frac{\lambda m}{\mu c}=\psi(0)
$$

When $c<\lambda m / \mu, \mathcal{B}_{m+1}\left(\tilde{Q}_{m}\right)=(1-\tilde{p})^{-1}$ where $\tilde{p}>1 /(m+1)$ and

$$
\tilde{p}(1-\tilde{p})^{m}=\frac{\lambda}{\mu c+\lambda}\left(\frac{\mu c}{\mu c+\lambda}\right)^{m} .
$$

Then

$$
\sum_{n=1}^{\infty} p_{n}(0)=\frac{\lambda}{(\lambda+\mu c)(1-\tilde{p})} \sum_{i=0}^{m-1}\left(\frac{\mu c}{(\lambda+\mu c)(1-\tilde{p})}\right)^{i}
$$

and straightforward manipulation using (6.9) gives $\sum_{n=1}^{\infty} p_{n}(0)=1$. The same argument applies when $c=\lambda m / \mu$.

\section{Concluding remarks}

As indicated earlier, there is only a small number of explicit formulae for the density function of the time of ruin or the probability function of the number of 
claims until ruin in Sparre Andersen models. Many of these have similar forms to the functions considered in this note. Consequently we can apply the ideas in this note to other cases. We have not tried to give an exhaustive list in this note; rather, we have sought to show how seemingly complicated expressions can easily be simplified if we can evaluate generalised binomial series.

\section{References}

[1] Dickson, D.C.M. (2012) The joint distribution of the time to ruin and the number of claims until ruin in the classical risk model. Insurance: Mathematics \& Economics 50, 334-337.

[2] Dickson, D.C.M., Hughes, B.D. and and Zhang, L. (2005) The density of the time to ruin for a Sparre Andersen process with Erlang arrivals and exponential claims. Scandinavian Actuarial Journal 2005, 5, 358-376.

[3] Dickson, D.C.M. and Li, S. (2010) Finite time ruin problems for the Erlang(2) risk model. Insurance: Mathematics \& Economics 46, 12-18.

[4] Dickson, D.C.M. and Qazvini, M. (2016) Gerber-Shiu analysis of a risk model with capital injections. European Actuarial Journal 6, 409-440.

[5] Drekic, S. and Willmot, G.E. (2003) On the density and moments of the time to ruin with exponential claims. ASTIN Bulletin 33, 11-21.

[6] Frostig, E., Pitts, S.M. and Politis, K. (2012) The time to ruin and the number of claims until ruin for phase-type claims. Insurance: Mathematics $\mathcal{E}$ Economics 51, 19-25.

[7] Gerber, H.U. (1988) Mathematical fun with the compound binomial process. ASTIN Bulletin 18, 161-168.

[8] Graham, R.L., Knuth, D.E. and Patashnik, O. (1994). Concrete Mathematics, 2nd edition. Addison-Wesley, Upper Saddle River, NJ.

[9] Grandell, J. (1991) Aspects of risk theory. Springer-Verlag, New York.

[10] Jain, G.C. and Consul, P.C. (1971) A generalized negative binomial distribution. SIAM Journal of Applied Mathematics 21, 501-513.

[11] Landriault, D., Shi, T. and Willmot, G.E. (2011) Joint densities involving the time to ruin in the Sparre Andersen risk model under exponential assumptions. Insurance: Mathematics and Economics 49, 371-379.

[12] Li, S. and Sendova, K.P. (2013) The finite-time ruin probability under the compound binomial risk model. European Actuarial Journal 3, 249-271.

[13] Nie, C., Dickson, D.C.M. and Li, S. (2015) The finite time ruin probability in a risk model with capital injections. Scandinavian Actuarial Journal 2015, 301-318. 
[14] Sparre Andersen, E. (1957) On the collective theory of risk in the case of contagion between the claims. Transactions of the $X V$ International Congress of Actuaries 2, 219-229.

[15] Zhao, C. and Zhang, C. (2013) Joint density of the number of claims until ruin and the time to ruin in the delayed renewal risk model with $\operatorname{Erlang}(n)$ claims. Journal of Computational and Applied Mathematics 244, 102-114. 